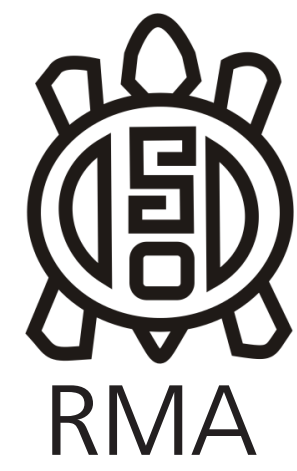

Dossier

\section{Enfermedad degenerativa articular en la población elite y no elite del sitio arqueológico Solcor-3: periodo medio, San Pedro de Atacama, norte de Chile}

\author{
Degenerative joint disease in the elite and non-elite population of \\ Solcor-3 archaeological site: middle period, San Pedro de Atacama, \\ north of Chile
}

Viviana. B. Llagostera Leyton*

* Facultad de Cs. Sociales y Jurídicas, Universidad de Tarapacá, Arica - Chile. E-mail:v.llagostera@gmail.com

\begin{abstract}
Resumen
Solcor-3 es una población atacameña que vivió durante el periodo Agroalfarero Medio caracterizada, según sus contextos culturales, por integrar dos grupos con diferencias en su estatus social. Los análisis realizados estuvieron enfocados a determinar si había desigualdad en su calidad de vida, desde el punto de vista de la enfermedad degenerativa articular (EDA). Se pudo determinar que, el estatus social no estaría relacionado con el desarrollo de esta patología, a excepción de los individuos femeninos quienes presentan una mayor prevalencia de la enfermedad, principalmente en sus extremidades inferiores (cadera derecha 50\% y rodilla derecha 18,5\%). Estos resultados serían correspondientes con actividades de pastoreo y agricultura, sin descartar otros factores etiológicos que afectarian al género femenino.
\end{abstract}

Palabras clave: periodo Medio; San Pedro de Atacama; enfermedad articular degenerativa; actividad corporal; estatus social.

\begin{abstract}
Solcor-3 is an atacameño population that lived during the Middle Period, which is characterised, according to its cultural context, by the inclusion of two different social groups based on their social status. The focus of the analyses were to determine if there were life inequalities, through the study of degenerative joint disease (DJD). It was possible to determine that social status is not related to the development of this pathology, except for female individuals, who presented higher prevalences of degenerative joint disease mainly in their lower extremities (right hip 50\% and right knee 18,5\%). These results are in accordance with grazing and agricultural activities, although DJD in females could also be influenced by other etiological factors.
\end{abstract}

Keywords: Middle period; San Pedro de Atacama; degenerative joint disease; body activity; social status.

En la región norte del territorio chileno, específicamente en la Puna Salada entre la Cordillera de los Andes y la Cordillera de Domeyko se ubica el oasis de San Pedro de Atacama (Figura 1). Este escenario geográfico permitió el sustento de los primeros habitantes hace más de 9.000 años A.C. junto al posterior desarrollo y consolidación de la Cultura San Pedro (Llagostera 2004; Núñez 2007).

Debido a la excelente preservación de los restos culturales como bioantropológicos, se ha podido reconocer a los antiguos atacameños como una población que basó su subsistencia en el desarrollo de la agricultura y el pastoreo de camélidos, a partir del cual derivaron actividades como el tráfico caravanero que permitió el intercambio de bienes con otras regiones de los Andes Centro-Sur (Llagostera 2004; Núñez 2007).
Respecto de la calidad de vida de estos antiguos atacameños, las investigaciones diacrónicas y comparativas entre los Periodo Medio (400-1000 D.C.) e Intermedio Tardío (1000-1450 D.C), han planteado que la calidad de vida, durante el primer periodo habría sido beneficiosa para indicadores como dimorfismo sexual y dieta (Costa 1988; Costa y Llagostera 1994; Costa et al. 2004; Neves y Costa 1998; 1999), mientras que otras investigaciones, relacionadas con tensión social, infecciones y salud dental darían cuenta de un escenario poco saludable para el periodo (Hubbe et al. 2012; Lessa 1999; Torres-Rouff 2002; Torres-Rouff y Costa 2005; Tótora da Gloria et al. 2011; Varela et al. 1990).

Entre los distintos periodos del desarrollo cultural de la región atacameña, el periodo Agroalfarero Medio (400- 


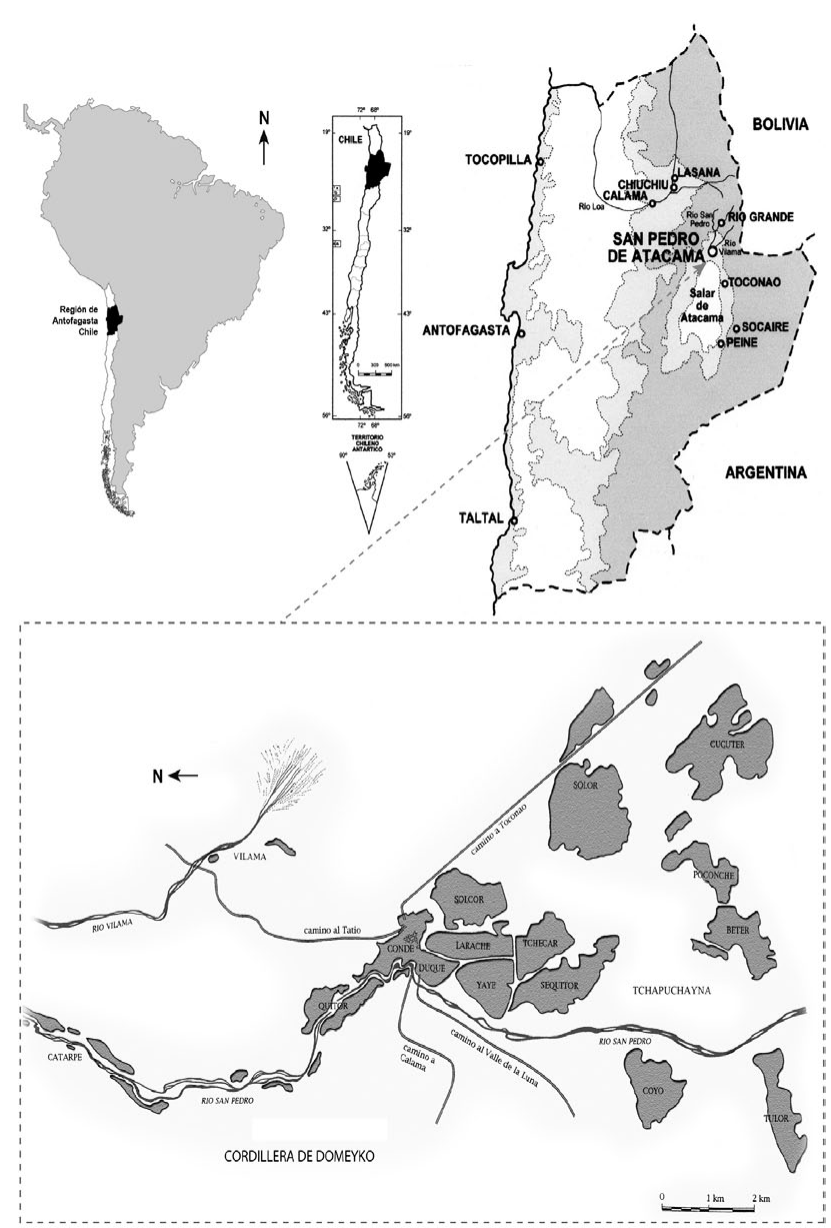

Figura 1. Localización de la colección en estudio.

Figure 1. Location of the studied collection.

1000 D.C.) se destacó, por ser una de las épocas en que se dieron los cambios más importantes a nivel social y económico, ya que San Pedro de Atacama se convirtió en un núcleo central de la vasta red altiplánica proveniente de Tiwanaku, consolidando aún más su interacción regional de intercambio de bienes económicos y suntuarios (Berenguer et al. 1986; Llagostera 2004). Fue gracias a esta actividad que una parte de la población pudo optar a una mayor diversidad de bienes (Llagostera 1996), lo que a su vez habría generado cierta desigualdad social que favoreció la salud y prosperidad de este segmento de la población (Hubbe et al. 2012).

Sin embargo, hasta ahora no está claro si la diferencia de calidad de vida y estatus social observada dentro de este periodo, es reconocida también desde el punto de vista funcional del cuerpo como consecuencia del estrés de las actividades habituales. Para explorar la intensidad de los niveles de exigencia corporal o estrés mecánico sufrido por los individuos, nos centraremos en el estudio de la enfermedad degenerativa articular (EDA) del esqueleto apendicular. Por lo tanto, esta condición patológica será relacionada con la diferenciación social registrada en la antigua población atacameña de Solcor-3, perteneciente al periodo Agroalfarero Medio en San Pedro de Atacama (Bravo y Llagostera 1986).

\section{Desarrollo cultural y el sitio arqueológico de Solcor-3}

La prehistoria cultural de San Pedro de Atacama cuenta con fechas tempranas de hace unos 9000 años A.C. Desde entonces, bajo un régimen de trashumancia, el hombre desarrolló actividades de subsistencia basados en la caza y recolección, pasando progresivamente al sedentarismo junto con las primeras actividades hortícolas, el manejo de camélidos silvestres a domésticos y la complejización social (Agüero 2005; Berenguer et al. 1986; Llagostera 2006; Núñez 2007; Núñez y Santoro 2011).

Fue durante el periodo Agroalfarero Medio (400 a 1000 D.C.) en donde se habría generado la consolidación de la influencia de Tiwanaku, dentro de la región atacameña (Tarragó 1989), documentándose arqueológicamente un mayor crecimiento poblacional y aumento de las ofrendas funerarias en cantidad y calidad (Llagostera 1996, 2004;Tarragó 1989; Uribe y Agüero 2001). Es durante este tiempo que San Pedro de Atacama asume un rol fundamental, transformándose en una localidad con un sistema eficaz de contacto y circulación de toda clase de bienes y productos, gracias a la consolidación de las redes caravaneras. Este tipo de actividades y la interacción con regiones vecinas, habrían generado la mayor estabilidad social y económica vivida por los atacameños en su desarrollo precolombino (Llagostera 1996, 2004).

Sin embargo, desde el punto de vista de la salud poblacional, este panorama habría sido algo distinto, ya que las últimas investigaciones nos presentan una perspectiva variada, no muy beneficiosa para toda la población (Hubbe et al. 2012; Nado et al. 2012; Tótora Da Gloria et al. 2011; Torres-Rouf 2011).

Durante el periodo Agroalfarero Medio hubo presencia de algunos individuos foráneos, junto a un sostenido aumento poblacional (Nado et al. 2012; TorresRouff y Knudson 2007; Varela y Cocilovo 2011). Lo cual, sería concordante con los mayores niveles de infecciones inespecíficas detectadas durante este periodo (50\% población adulta), posiblemente por causa del empeoramiento del entorno sanitario producto de la agregación social (Tótora Da Gloria et al. 2011).

Respecto al uso funcional del cuerpo, los análisis de osteofitos de columna vertebral, detectaron la mayor prevalencia (ca. 20\%) y los mayores grados promedios en el segmento torácico inferior $(1,02)$ y el lumbar $(1,48)$. Resultados que se relacionarían con actividades de carga sobre la espalda. Esto indicaría que durante el Periodo Medio, Tiwanaku habría contribuido a generar mayor desarrollo de las actividades de subsistencia comparado con el Periodo Intermedio Tardío (López-Barrales et al. 2015).

Sin embargo, las investigaciones de tensión social, ha planteado que durante este periodo hubo una alta prevalencia de traumas corporales (47\%) en comparación 
con el periodo Tardío (Lessa y Mendonça de Souza 2004, 2006). Además, la presencia de algunos individuos con mejores índices en la dieta (Hubbe et al. 2012), baja frecuencia de lesiones craneanas y contextos funerarios más abundantes y de calidad (Torres-Rouff 2011), sugieren la existencia de una elite atacameña(Hubbe et al. 2012; Torres-Rouff 2011). Por lo tanto, estas evidencias proponen el surgimiento de conflictos interpersonales por consecuencia de la nueva influencia ideológica y económica que habría traído consigo la influencia Tiwanaku, favoreciendo a sólo algunos miembros de la sociedad atacameña, incrementando consecuentemente, la desigualdad social (Lessa y Mendonça de Souza 2004, 2006).

\section{El sitio arqueológico Solcor-3}

Solcor-3 es un cementerio que se ubica en el sector oriente del núcleo de los oasis de San Pedro de Atacama, excavado en la década de los 80. Los trabajos de campo expusieron 93 tumbas con un total de 153 cuerpos, entre hombres, mujeres y niños, en condiciones esqueletizadas o bien en fardos funerarios, quienes fueron depositados en posición sentada y flexionados (Bravo y Llagostera 1986). La secuencia ocupacional del sitio fue determinada a través de observaciones en planta, fechados por termoluminiscencia, radio-carbónicos y por la distribución de los patrones tipológicos cerámicos presentes en las tumbas. De acuerdo a esto, Bravo y Llagostera (1986) establecieron dos ocupaciones, una temprana (250 años A.C. a 480 años D.C.), correspondiente a fines del periodo Agroalfarero Temprano, y otra posterior (480 a 920 años D.C.), propia del periodo Agroalfarero Medio.

Posteriormente, nuevas investigaciones y reevaluación de fechados (Hubbe et al. 2012; Torres-Rouff 2011) determinaron que los dos componentes serían contemporáneos, pero diferenciados por desigualdad social, distinguiendo grupos elite y no elite (433 a 1176 años cal D.C). Los individuos del grupo elite serían aquellos cuyo ajuar funerario destaca por poseer objetos relacionados con Tiwanaku y cerámicas foráneas, mientras que los individuos de grupo no elite evidencian la falta de objetos tiwanakotas junto a ceramicos locales (Hubbe et al. 2012).

\section{Enfermedad Degenerativa Articular}

Dentro del análisis de los restos esqueletales, en las poblaciones prehistóricas, se considera la Enfermedad Degenerativa Articular (EDA) como una patología crónica y degenerativa caracterizada por la pérdida gradual del cartílago articular, siendo la enfermedad más común en todas las poblaciones (Kennedy 1989; Larsen 1999; Peña y Fernández 2007; Waldron 2009). Hoy se reconocen una serie de factores posibles que tienden a precipitar el deterioro estructural o daño degenerativo de una articulación (Steckel y Rose 2002; Weiss y Jurmain 2007). Entre ellos se identifican la edad, la genética, el sexo, los traumas y el más importante: el movimiento debido a los efectos acumulativos del estrés mecánico (Steckel y Rose 2002; Waldron 2009; Yousuf 1978).

Esta patología osteoarticular ha sido vinculada al estilo de vida, ya que se interpreta como el relato corporal de la actividad humana en el medio ambiente, producto del estrés producido por los movimientos acumulativos y repetitivos requeridos en las actividades físicas (Larsen 1999). Estas actividades habituales provocan tensión constante sobre las articulaciones, transformándose a la larga en un proceso dinámico y gradual de desgaste fisiológico de los componentes articulares expuestos al estrés (Bridges 1992; Furey 2002; Jurmain 1977; Lieverse et al. 2007; Mow y Hung 2004; Steckel y Rose 2002; Steinbock 1976).

Las actividades habituales implican la intervención de varias fuerzas, tanto longitudinales y tangenciales que proceden del exterior, como de las contracciones musculares y fuerzas compresivas no fisiológicas de impactos sobre el hueso subcondral, que causan microfracturas (Cailliet 2006). Cualquiera de estas fuerzas produce trastornos en la anatomía y biomecánica articular, alterando la alineación de las carillas articulares e influyendo, consecuentemente, en la distribución de las fuerzas y cargas anatómicas, provocándose así, el inicio del daño estructural del cartílago articular (Weiss y Jurmain 2007). Si este daño sigue en progreso, consecuentemente, el tejido óseo responderá con la formación de porosidad en la superficie articular, hueso nuevo en las áreas subcondrales y márgenes articulares (lipping) y en etapas más avanzadas; eburnación o pulimiento de la carilla articular más afectada (Furey 2002; Waldron 2009).

\section{Materiales y Métodos}

Los análisis fueron realizados sobre una muestra de 49 individuos completos adultos, pertenecientes al cementerio de Solcor-3, resguardados en los depósitos del Instituto de Arqueología y Antropología del Museo R.P. Gustavo Le Page en San Pedro de Atacama (Tabla1). Solcor-3 cuenta con dos grupos de individuos contemporáneos diferenciados según sus contextos culturales, en un grupo no elite de 31 individuos y 18 pertenecientes a contextos elite (Hubbe et al. 2012;

\begin{tabular}{cccccccc}
\hline & \multicolumn{1}{c}{ Masculinos } & \multicolumn{3}{c}{ Femeninos } & Total \\
\hline Solcor-3 & $20-34$ & $35-45$ & $>45$ & $20-34$ & $35-45$ & $>45$ & \\
\hline No Elite & 11 & 5 & 3 & 4 & 6 & 2 & 31 \\
Elite & 6 & 2 & 1 & 4 & 3 & 2 & 18 \\
\hline Total & $\mathbf{1 7}$ & $\mathbf{7}$ & $\mathbf{4}$ & $\mathbf{8}$ & $\mathbf{9}$ & $\mathbf{4}$ & $\mathbf{4 9}$ \\
\hline
\end{tabular}

Tabla 1. Muestras óseas para el análisis de EDA, según rangos de edad, estatus social y sexo.

Table 1. Skeletal series studied by age, status social and sex. 
Torres-Rouf 2011).

Para la identificación de sexo fue considerada la morfología pélvica y craneana, la estimación de edad de acuerdo a los criterios de los signos de maduración de la sínfisis pubiana, del ilium, el desgaste dentario y el grado de sinostosis de las suturas craneanas, de acuerdo a los criterios empleados por Gilbert y Mckern (1973), Lovejoy et al. (1985), Lovejoy (1995), McKern y Stewart (1957) y Meind y Lovejoy (1985). De acuerdo a esto se establecieron intervalos etarios que involucraron los siguientes rangos de edad: 20-34 años; 35-44 años y mayores de 45 años.

Los indicadores y determinación del grado de severidad de la EDA fueron evaluados de acuerdo a la información organizada en el Manual de Procedimientos y Análisis Osteológico confeccionado por Neves, Costa y Santoro (1988). Basado en Bass (1971) y Ubelaker (1978): (0) Ausencia de signos degenerativos; (1) grado leve: observación en la carilla articular de porosidad y formación de lipping; (2) grado moderado: se observa el $50 \%$ del área afectada de la articulación e inicio de rasgos de eburnación; (3) grado severo: se observa más del 50\% de la carilla articular afectada, presencia de porosidad, lipping y eburnación y (4) grado extremo: se observa la superficie articular desfiguradas, junto con signos de eburnación y anquilosis.

El análisis tomó en cuenta sólo las articulaciones apendiculares de hombros, codos, muñecas, caderas, rodillas y tobillos (Tabla 2 ), considerando, rango etario, sexo, lateralidad y grupo social (elite y no elite). Debido a que contábamos con individuos de distintos rangos etarios (20 a >45 años), se comparó la frecuencia de la enfermedad tanto por sexo como por grupos elite $y$ no elite, usando la prueba $X^{2}$ de Pearson (corrección de Yates), para determinar si la edad estaba asociada a la presencia de EDA.

La prevalencia de la EDA fue determinada dividiendo el número de individuos afectados por el número del total de individuos observados (n/N-100). Además, con el fin de conocer si existía diferencia entre los grupos, se usó el valor de la mediana obtenida según el grado registrado por cada carilla articular. Este valor, entendido como una medida de tendencia central, reflejaría el grado de compromiso articular, asumiendo que la articulación fue afectada en su totalidad. El valor final fue usado en la prueba no paramétrica de U de Mann Whitney.

\section{Resultados}

Para determinar si la edad tenía influencia en las diferencias observadas se compararon los tres grupos de rangos etarios propuestos, distinguiendo según el estatus social. Observe que el grupo que se encuentra afectado por el mayor la mayor frecuencia de la enfermedad, corresponde al grupo etario entre 35 a 44 años de edad pertenecientes al grupo No elite. Sin embargo, no se encontraron diferencias significativas entre las categorías etarias (Tabla 3). Por lo tanto, la edad no se relacionaría con la presencia de EDA en el esqueleto apendicular.

El análisis de la prevalencia de la enfermedad determinó que los individuos pertenecientes al grupo No Elite y Elite presentan las frecuencias más altas de la EDA en el hombro derecho con un 53,84 \% y 52,94\% respectivamente (Tabla 4). Al comparar la enfermedad entre el grupo de hombres y mujeres en la población general, se observó que el tanto el hombro derecho de los hombres (48\%) como esta articulación en las mujeres (61\%) tienen los valores más altos de la enfermedad, seguidos por la articulación de cadera derecha en la población femenina alcanzando el 50\% (Tabla 5).

Es así como el análisis estadístico de prueba no paramétrica, permitió comparar, por un lado la EDA entre la población elite y no elite $y$, por otro, entre ambos sexos. Si bien el análisis de prevalencia resultó alto para las articulaciones superiores del lado derecho, estos valores no fueron significativos (con un nivel error del 5\%) entre los grupos con distinción social, ni entre los sexos. De la misma manera, se realizó con el resto de las articulaciones comparando entre el grupo elite y no elite, no observándose diferencias significativas para ninguna articulación específica (Tabla 6). Sin embargo, al comparar en general a la población masculina con la femenina, se obtuvieron diferencias estadísticamente significativas, específicamente relacionadas con la articulación de cadera derecha (valor $p=0,025$ ) y rodilla derecha (valor $p=0,033$ ). La observación de las frecuencias realizadas con anterioridad, nos permitió determinar que el mayor desarrollo de la EDA se presentó en el grupo de las mujeres.

\section{Discusión}

Según los resultados obtenidos, podemos determinar que se no encontraron diferencias en el desarrollo de la EDA entre los grupos con distinción de estatus social,

\begin{tabular}{|c|c|}
\hline Articulación & Áreas observadas \\
\hline Hombro & Cavidad gleonoidea y cabeza de húmero. \\
\hline Codo & Capitulum, tróclea, cabeza del radio, fosa radial, incisura troclear. \\
\hline Muñeca & Incisura ulnar, carpo-ulnar, carpo-radial. \\
\hline Cadera & Acetábulo y cabeza de fémur. \\
\hline Rodilla & $\begin{array}{c}\text { Cóndilos femorales, cara patelar, cóndilos tibiales, cara articular de fíbula, carillas interna y } \\
\text { externa de la patela. }\end{array}$ \\
\hline Tobillo & $\begin{array}{c}\text { Cara articular inferior de la tibia, carilla tibio-fibular, maleolo lateral, maleolo medial, tróclea } \\
\text { del astrágalo y carilla articular maleolar lateral y medial. }\end{array}$ \\
\hline
\end{tabular}

Tabla 2. Articulaciones evaluadas.

Table 2. Joints studied. 
Tabla 3. Resultados de Chi cuadrado (corrección de Yates), relación entre individuos afectados con EDA y edad. Rangos de 20 a 24 años, 35 a 44 años y mayores de 45 años.

Table 3. Chi square results (Yates correction), relationship between DJD and age: 20 to 24 years, 35 to 44 years and over 45 years.

\begin{tabular}{|c|c|c|c|c|c|c|}
\hline \multicolumn{7}{|c|}{ Edad } \\
\hline & $20-34$ & $35-44$ & $>45$ & Total & $x^{2}$ & valor $p$ \\
\hline No afectados & $12(50 \%)$ & $3(16.7 \%)$ & $2(28.6 \%)$ & $17(34.7 \%)$ & \multirow{3}{*}{5.179} & \multirow{3}{*}{0.075} \\
\hline & & & & & & \\
\hline Afectados & $12(50 \%)$ & $15(83.3 \%)$ & $5(71.4 \%)$ & $32(65.3 \%)$ & & \\
\hline \multirow[t]{2}{*}{ Total } & $24(100 \%)$ & $18(100 \%)$ & $7(100 \%)$ & $49(100 \%)$ & & \\
\hline & \multicolumn{2}{|l|}{ No Elite } & \multicolumn{2}{|l|}{ Elite } & & \\
\hline Edad & Afectados & No afectados & Afectados & No afectados & $x^{2}$ & Valor $p$ \\
\hline $20-34$ & 7 & 8 & 5 & 4 & 0.18 & 1.000 \\
\hline $35-44$ & 10 & 1 & 5 & 2 & 0.19 & 0.665 \\
\hline$>45$ & 3 & 2 & 2 & 0 & 0.02 & 0.894 \\
\hline
\end{tabular}

Tabla 4. Prevalencia de la EDA distribuida según el estatus social. n:casos afectados, N: casos observados.

Table 4. DJD prevalence by social status.n: cases affected, $N$ : cases observed.

\begin{tabular}{ccccccccc}
\hline \multirow{2}{*}{ Articulaciones } & \multicolumn{3}{c}{ Solcor-3 } & No elite & \multicolumn{4}{c}{ Solcor-3 Elite } \\
\cline { 2 - 9 } & \multicolumn{2}{c}{ Izquierdo } & \multicolumn{2}{c}{ Derecho } & \multicolumn{2}{c}{ Izquierdo } & Derecho \\
\cline { 2 - 9 } & $\mathrm{n} / \mathrm{N}$ & $\%$ & $\mathrm{n} / \mathrm{N}$ & $\%$ & $\mathrm{n} / \mathrm{N}$ & $\%$ & $\mathrm{n} / \mathrm{N}$ & $\%$ \\
\hline Hombro & $10 / 28$ & 35.71 & $14 / 26$ & 53.84 & $5 / 18$ & 27.77 & $9 / 17$ & 52.94 \\
Codo & $12 / 29$ & 41.37 & $10 / 27$ & 37.03 & $4 / 18$ & 22.22 & $7 / 17$ & 41.17 \\
Muñeca & $3 / 28$ & 10.71 & $4 / 28$ & 14.28 & $2 / 18$ & 11.11 & $3 / 16$ & 18.75 \\
Cadera & $11 / 29$ & 37.93 & $11 / 28$ & 39.28 & $3 / 18$ & 16.66 & $4 / 17$ & 23.52 \\
Rodilla & $3 / 29$ & 10.34 & $3 / 23$ & 13.04 & $0 / 18$ & 0 & $0 / 16$ & 0 \\
Tobillo & $1 / 30$ & 3.33 & $0 / 30$ & 0 & $0 / 16$ & 0 & $1 / 16$ & 6.25 \\
\hline
\end{tabular}

Tabla 5. Prevalencia de la EDA distribuida según sexos. n:casos afectados, N: casos observados.

Table 5. DJD prevalence by sex.n: cases affected, N: cases observed.

\begin{tabular}{ccccccccc}
\hline \multirow{3}{*}{ Articulaciones } & \multicolumn{4}{c}{ Masculinos } & \multicolumn{4}{c}{ Femeninos } \\
\cline { 2 - 9 } & \multicolumn{2}{c}{ Izquierdo } & \multicolumn{2}{c}{ Derecho } & \multicolumn{2}{c}{ Izquierdo } & Derecho \\
\cline { 2 - 9 } & $\mathrm{n} / \mathrm{N}$ & $\%$ & $\mathrm{n} / \mathrm{N}$ & $\%$ & $\mathrm{n} / \mathrm{N}$ & $\%$ & $\mathrm{n} / \mathrm{N}$ & $\%$ \\
\hline Hombro & $6 / 25$ & 24 & $12 / 25$ & 48 & $9 / 20$ & 45 & $11 / 18$ & 61.11 \\
Codo & $7 / 27$ & 25.92 & $9 / 26$ & 34.61 & $9 / 20$ & 45 & $8 / 18$ & 44.44 \\
Muñeca & $3 / 27$ & 11.11 & $2 / 27$ & 7.40 & $2 / 19$ & 10.52 & $5 / 17$ & 29.41 \\
Cadera & $6 / 27$ & 22.22 & $6 / 27$ & 22.22 & $8 / 20$ & 40 & $9 / 18$ & 50 \\
Rodilla & $1 / 28$ & 3.57 & $0 / 23$ & 0 & $2 / 19$ & 10.52 & $3 / 16$ & 18.50 \\
Tobillo & $0 / 28$ & 0 & $0 / 28$ & 0 & $1 / 18$ & 5.55 & $1 / 18$ & 5.55 \\
\hline
\end{tabular}

El hecho de que el mayor desarrollo de EDA haya sido significativo en el grupo total de las mujeres sin tener distinción de grupo estatus social, específicamente en la cadera y rodilla derecha, nos podría sugerir una posible diferenciación de género, sumado al factor genético y biomecánico como variables de predisposición a la enfermedad (Weiss y Jurmain 2007).

Respecto al primer caso, dentro de las actividades físicas implicadas con el desarrollo de EDA de cadera se encuentra la agricultura (Peña y Fernandez-Lopez et al. 2007). De igual manera, no podemos descartar que esta patología, también se relacione con una mayor movilidad, generada por caminatas extensas, relacionadas con el intercambio y/o actividades de tráfico caravanero, como fue propuesto por Pomeroy (2013).

Las antiguas mujeres atacameñas estarían involucradas en actividades físicas que habrían causado el desarrollo de la enfermedad; como prolongadas y repetidas flexiones de las articulaciones involucradas, posiciones mantenidas de hiperflexión de ni entre el sexo de los individuos con el estatus social. Por lo tanto podemos decir que, en general, el grupo de menor estatus social no estaría sometido a mayor demanda de actividad física que el grupo Elite. Este planteamiento sería coherente con las investigaciones relacionadas al estrés funcional de la columna vertebral realizadas para la misma colección (López-Barrales et al. 2015). A su vez, esto respalda la propuesta de Nado et al. (2012), quienes sugieren que la diferenciación social en los contextos funerarios del Periodo Medio en San Pedro de Atacama, estaría relacionada con la incorporación de bienes por parte de un segmento de la población, como un mecanismo de distinción de identidad ideológica. Descartando así el sometimiento de un grupo sobre otro y sin tener influencia sobre el desarrollo de las actividades subsistencia económica.

La EDA es reconocida como una condición gradual que se relaciona con múltiples factores, entre ellos la edad (Bridges 1992). De acuerdo a nuestros resultados, en este caso, la edad no sería una variable determinante en el desarrollo de la enfermedad, sino más bien estaría relacionada con las actividades físicas repetitivas. cadera y rodilla para la actividad de molienda, caminatas por terrenos escabrosos o levantar cargas superiores a 25 kg (Arriaza y Standen 2008; Lieverse et al. 2007; Peña y Fernández-López 2007; Zhang et al. 2004). Relacionado a esto último, cabe mencionar, parte del relato de un actual poblador atacameño, quien recordando su niñez, refirió que antiguamente era labor de las mujeres ir a buscar agua. Para realizar esta tarea, debían bajar las quebradas llevando una gran vasija de cerámica, la que una vez llena, era apoyada y trasladada sobre una de sus caderas (Osvaldo Rojas comunicación personal 2016). Las investigaciones etnográficas del mundo andino, realizadas por Carrasco y Gavilán (2014) podrían aclarar mucho más el tema de la distinción de género en las poblaciones prehispánicas y dar respaldo a nuestra hipótesis. Las investigadoras han descrito una efectiva división sexual del trabajo dentro de la unidad familiar campesina, donde se observa mayor privilegio para los hombres y recarga de trabajo físico para las mujeres. Es en ellas donde descansan las labores reproductivas (alimentación, crianza de hijos, aseo, lavado) y productivas, como la siembra y el aporcamiento en la agricultura, el pastoreo, el hilado fino y el tejido (Gavilán 2002). Por lo tanto, la mujer 


\begin{tabular}{|c|c|c|c|c|c|c|}
\hline & \multicolumn{2}{|c|}{ Solcor-3 No Elite } & \multicolumn{2}{|c|}{ Solcor-3 Elite } & \multirow[b]{2}{*}{$\begin{array}{l}\text { U de Mann } \\
\text { Whitney }\end{array}$} & \multirow[b]{2}{*}{ valor $p$} \\
\hline & $\mathrm{N}$ & $\sum$ de rangos & $\mathrm{N}$ & $\sum_{\text {rangos }}$ de & & \\
\hline Hombro Der. & 26 & 571.50 & 17 & 374.50 & 220.500 & 0.989 \\
\hline Hombro Izq. & 28 & 677.50 & 18 & 403.50 & 232.500 & 0.595 \\
\hline Codo Der. & 27 & 591.00 & 17 & 399.00 & 213.000 & 0.649 \\
\hline Codo Izq. & 29 & 749.00 & 18 & 379.00 & 208.000 & 0.168 \\
\hline Muñeca Der. & 28 & 620.50 & 16 & 369.50 & 214.500 & 0.715 \\
\hline Muñeca Izq. & 28 & 656.50 & 18 & 424.50 & 250.500 & 0.950 \\
\hline Cadera Der. & 28 & 685.50 & 17 & 349.50 & 196.500 & 0.244 \\
\hline Cadera Izq. & 29 & 752.00 & 18 & 376.00 & 205.000 & 0.127 \\
\hline Rodilla Der. & 23 & 484.00 & 16 & 296.00 & 160.000 & 0.138 \\
\hline Rodilla Izq. & 29 & 723.00 & 18 & 405.00 & 234.000 & 0.163 \\
\hline Tobillo Der. & 30 & 690.00 & 16 & 391.00 & 225.000 & 0.171 \\
\hline Tobillo Izq. & 30 & 713.00 & 16 & 368.00 & 232.000 & 0.465 \\
\hline
\end{tabular}

Tabla 6. Resultados de la prueba de $U$ de Mann Whitney, comparando la mediana de la EDA entre estatus social y sexo, distribuidos según lateralidad.

Table 6. U Mann Whitney test result, comparisons of DJD median, between social status and sex, distributed by laterality.

actividades habituales. Si a esto se suma, la inmigración caravanera de algunos de los miembros masculinos del núcleo familiar, sus actividades tendrían que ser suplidas por otros miembros del grupo local y por tanto, éstos tendrían que trabajar con mayor intensidad y por más tiempo, condiciones que podrían explicar, en parte, la disminución de la calidad de vida desde el punto de vista funcional del cuerpo de las mujeres.

\section{Conclusión}

El análisis de la enfermedad degenerativa en cumpliría un rol activo dentro de la unidad doméstica, realizando sus actividades y asistiendo constantemente las labores masculinas, situación que se ve agravada en caso de distanciamiento o migración temporal de los varones (Carrasco y Gavilán 2014; Gavilán 2002).

Por su parte el factor genético tiene una alta heredabilidad que afectaría al sexo femenino, cerca del 50\% y específicamente a la articulación de rodilla en un 39\% (Peña y Fernández-López 2007). Por otro lado, la fisiología y biomecánica articular femenina, pueden predisponer al desarrollo de la enfermedad, ya que por ejemplo, el aumento de estrógenos en la mujer, causa laxitud de ligamentos y un mayor ancho de la pelvis, favoreciendo la tendencia a rodillas en valgo (articulación femoro-tibial desviada a la línea media). Ambos factores, en conjunto o por separado, predisponen a una importante inestabilidad y movilidad anómala articular, afectando finalmente, la distribución de la carga articular (Millares y Puig 1998).

Estamos de acuerdo con que la influencia de Tiwanaku conllevó a la sociedad atacameña a cambios sustanciales (Costa et al. 2004; Llagostera 1996; 2004; Uribe y Agüero 2001), transformaciones que pudieron haber beneficiado sólo a algunos individuos y a ciertos rasgos de la calidad de vida (Hubbe et al. 2012; Torre-Rouff 2011). No obstante, estos cambios, no afectaron las bases de la subsistencia (agricultura y pastoreo), sino más bien, creemos que estas actividades se habrían reforzado o intensificado, generando mayor nivel de actividad física dentro del estamento femenino de la población de Solcor-3.

Es posible que durante el periodo Medio, la mayor agregación de personas provenientes de otras localidades, haya provocado aumento en la demanda de recursos alimenticios locales, debiéndose aumentar la mano de obra atacameña y en consecuencia, intensificar algunas

el esqueleto apendicular de antiguas poblaciones, si bien no es un indicador preciso de actividades culturalmente pautadas, nos permite un acercamiento a la inferencia de comportamientos y posturas relacionadas con la actividad corporal. A su vez, y dependiendo de la severidad de las lesiones, podemos deducir una buena o mala calidad de vida biológica en estos habitantes, es decir cuanto más se exige a una parte del cuerpo en el desempeño de las actividades diarias, mayor es la posibilidad de desarrollar esta enfermedad degenerativa de origen funcional en las articulaciones involucradas (Buikstra y Ubelaker 1994).

En el caso de los individuos de Solcor-3, se determinó que la edad no es un factor concluyente en el deterioro articular y por tanto, las lesiones encontradas podrían ser atribuidas a actividades físicas repetitivas, sin descartar factores genéticos y biomecánicos, especialmente en el caso del grupo femenino.

En general no obtuvimos resultados que nos relacionen directamente estatus social con el desarrollo de estrés corporal. Así como tampoco se relacionó la distribución sexual entre ambos grupos de estatus social. Sin embargo, los individuos femeninos de la colección, si estaban significativamente más afectadas que el resto de los individuos. Esto nos sugieren un posible sistema de división de tareas de género desarrollada en la antigua población de Solcor-3, donde el daño articular tendría correspondencia con actividades de subsistencia basada en una economía de pastoreo y labores agrícolas, comprometiendo en forma directa el trabajo de las extremidades inferiores y por tanto, una deficiente calidad de vida, desde el punto de vista funcional del uso corporal del estamento femenino en comparación con los individuos masculinos del sitio de Solcor-3.

Santiago de Chile, 24 de Agosto 2017 


\section{Agradecimientos}

Agradecemos a Mario Henríquez, al Dr. Agustín Llagostera, Dr. Mark Hubbe, Ariadna Cifuentes y a Josefina Urrea por el tiempo dedicado a la revisión de este manuscrito, junto Fernando Castillo por la edición de imagen y a María Antonieta Costa, quien puso a disposición el banco de datos para la información utilizada en este trabajo y a los evaluadores anónimos del artículo, quienes aportaron con sus valiosas sugerencias.

\section{Bibliografía}

Agüero, C. 2005. Aproximación al asentamiento humano temprano en los oasis de Atacama. Estudios Atacameños, 30: 29-60.

Arriaza, B., V. Standen. 2008. Bioarqueología, historia biocultural de los antiguos pobladores del extremo norte de Chile. Editorial Universitaria, Chile.

Berenguer, J., A. Deza, A. Román y A. Llagostera. 1986. La secuencia de Myriam Tarragó para San Pedro de Atacama: Un test por termoluminiscencia. Revista Chilena de Antropología, 5:17-54.

Bravo, L., A. Llagostera. 1986. Solcor-3: Un aporte al conocimiento de la Cultura San Pedro. Período 500 al 900 DC.Chungara, 16-17: 323-332.

Bridges P. 1992. Prehistoric arthritis in the Americas. Annual Review of Anthropology, 21:67-91.

Buikstra, J., D. Ubelaker. 1994. Standards for data collection from human skeletal remains. Arkansas Archaeological Survey Research Series 44, Estados Unidos.

Cailliet, R. 2006. Anatomía funcional, Biomecánica. Marban, España.

Carrasco, A.M, V. Gavilán. 2014. Género y etnicidad. Ser hombre y ser mujer entre los Aymaras del Altiplano chileno. Dialogo Andino, 45: 169-180.

Costa, M.A. 1988. Reconstitución física y cultural de la población tardía del cementerio Quitor-6 (San Pedro de Atacama). Estudios Atacameños, 9: 99-126.

Costa, M.A., A. Llagostera. 1994. Coyo-3: Momentos finales del periodo en San Pedro de Atacama. Estudios Atacameños, 11: 73-107.

Costa M.A., W.A. Neves y M. Hubbe. 2004. Influencia de Tiwanaku en la calidad de vida biológica de la población prehistórica de San Pedro de Atacama. Estudios Atacameños, 27: 103-116.

Furey, M. 2002. Joint lubrication. Schneck, D., D. Joseph, Biomechanics: principles and applications. 73-97. CRC Press, Londres.

Gavilán, V. 2002. Buscando Vida: hacia una teoría Aymara de la división de trabajo o por género. Chungara, 34: 101-117.
Gilbert, B.M., T. Mckern. 1973. A method for again the female of pubis. American Journal of physical Anthropology, 38:31-38

Jurmain, R. 1977. Stress and etiology of osteoarthritis. American Journal of Physical Anthropology, 46:353-366.

Hubbe, M., C. Torres-Rouff, W. Neves, L. King, P. Tótora DaGloria y M.A. Costa.2012. Dental health in northern Chile's Atacama oases: evaluating the Middle Horizon (AD 500-1000) impact on local diet. American Journal of Physical Anthropology, 148: $62-72$.

Kennedy, K. 1989. Skeletal markers of occupational stress. Iscan, M.Y., A. Kennedy y R. Liss, Reconstruction of life from the skeleton, 129-160. Alan R. Liss. Nueva York.

Larsen, C.S. 1999. Bioarchaeology, interpreting behavior from the human skeleton. Cambridge University press, Nueva York.

Lessa, A. 1999. "Estudios das lesoes traumaticas agudas como indicadores de tensao social na populacho do sitio-cementerio Solcor-3, SAP, Chile". Escola Nacional de saude Publica, fundacao Oswaldo Cruz. Tesis de Magíster en Salud Pública. Brasil.

Lessa, A., S. Mendoça De Sousa. 2004. Paleoepidemiologia dos traumatismos cotidianos em Solcor-3, San Pedro de Atacama, Chile: riscos diferenciados no período Tiwanaku? Antropología Portuguesa, 20-21: 183-207.

Lessa, A., S. Mendoça De Sousa. 2006. Broken noses for the gods: ritual battles in Atacama Desert during the Tiwanaku period. Memórias do Instituto Oswaldo Cruz, 101: 133-138.

Lieverse A., A. Weber, V. Baliiskiy, O. Goriunova y N. Savele. 2007. Osteoarthritis in Siberia's cis-Baikal: skeletal Indicators of hunter-gatherer adaptation and cultural change. American Journal of Physical Anthropology,132: 1-16.

Llagostera, A. 1996. San Pedro de Atacama: nodo de complementariedad reticular. Editado por X. Albo, M.I. Anatia, J. Hidalgo, L. Nuñez, A. Llagostera, M.I. Remy y B. Revesz. La integración surandina cinco siglos después.17-42, Centro de Estudios regionales Andinos "Bartolomé de las Casas", Cusco, Perú.

Llagostera, A. 2004. Los antiguos habitantes del salar de Atacama. Prehistoria atacameña. Editorial Pehuén y UCN. Santiago-Chile.

López- Barrales, R., M. Hubbe., E. Aspillaga., W. Neves Y H. Niemeyer. 2015. Osteofitosis vertebral en poblaciones prehispánicas de San Pedro de Atacama, Norte de Chile. Estudios Atacameños, 50: 177-194.

Lovejoy, C.O. 1995. Dental wear in the Libben population: Its functional pattern and role in the determination of adult skeletal age death. American Journal Physical Anthropology, 68: 47-56.

Lovejoy, C.O., R.S. Meindl, T.R. Pryzbeck. Y R.P Mensforth. 1985. Chronological metamorphosis of the auricular Surface of the ilium: a new method for the determination of adult skeletal age at death. American Journal of Physical Anthropology,68(1): 15-28. 
Mckern, T., T. Steward. 1957. Skeletal age changes in young American males, analyzed from the standpoint of identification. Technical Report EP-45, Natick, Massachusetts.

Meindl, R., C.O. Lovejoy. 1985. Ectocranial suture closure: a revised method or the determination of skeletal age at death base on the lateral-anterior sutures. American Journal of Physical Anthropology,68(1): 57-66.

Millares R., M. Puig. 1998. Sistema articular. Editores Millares y Puig. Biomecánica clínica del aparato locomotor,39-60, Editorial Masson S.A, Barcelona, España.

Mow V., C. Hung. 2004. Biomecánica del cartílago articular Editores M. Nordin y H. Frankel. Biomecánica básica del sistema musculo esquelético, 60-100, MacGraw Hill Interamericana, Madrid, España.

Nado, K., S. Marsteller S., L. King, B. Daverman, C. Torres-Rouff y K. Knudson. 2012. Examining local social identities through patters of biological and cultural variation in the Solcor ayllu, San Pedro de Atacama, Chile. Chungara, 44 (2): 341-357

Neves, W., M.A. Costa. 1998. Adult stature and standard of living in the prehistoric Atacama desert. Current Anthropology, 39 (2): 278-281.

Neves, W., M.A. Costa. 1999. Nutrition and quality of life in prehistoric San Pedro de Atacama, Chile. Antropología Física Latinoamericana, 2: 23-40.

Núñez, L. 2007. Vida y Cultura en el Oasis de San Pedro de Atacama. Editorial Universitaria, Santiago de Chile.

Núñez, L., C. Santoro. 2011. El tránsito Arcaico-Formativo en la circumpuna y valles occidentales del centro surandino: Hacia los cambios "neolíticos". Chungara,43 (1):487-530.

Peña, A., J. Fernández-López. 2007.Prevalencia y factores de riesgo de la osteoartritis. Reumatología Clínica, 3 (3): 6-12.

Pomeroy, E. 2013. Biomechanical insights into activity and long distance trade in the south-central Andes (AD 500-1450). Journal of Archaeological Science, 40(8): 3129-3140.

Steckel, R. y J. Rose. 2002. The backbone of history: health and nutrition in the western hemisphere. Cambridge University Press, Cambridge.

Steinbock, R.T. 1976. Paleopathological diagnosis and interpretation. Charles C. Thomas, Springfield.

Tarragó, M., 1989 “Contribución al conocimiento arqueológico de las poblaciones de los oasis de San Pedro de Atacama en relación con los otros pueblos puneños, en especial, el sector septentrional del valle Calchaquí". Universidad Nacional de Rosario Facultad de Humanidades y Artes. Tesis para optar al título de Doctor en Historia, especialidad Antropología. Rosario, Argentina.

Torres-Rouff, C.2002. Cranial vault modification and ethnicity in Middle Horizon San Pedro de Atacama, implications of environmental stress. Current Anthropology, 43(1):163-171.

Torres-Rouff, C. 2011. Hiding inequality beneath prosperity: patters of cranial injury in Middle Period San Pedro de Atacama, Northern Chile. American Journal of Physical Anthropology 146: 28-37.

Torres-Rouf, C., M.A. Costa. 2005. Interpersonal violence in prehistoric San Pedro de Atacama, Chile. American Journal of Physical Anthropology, 130(1):60-70.

Torres-Rouff, C., K. Knudson. 2007. Examining the life history of an individual from Solcor-3, San Pedro de Atacama: Combining bioarchaeology and archaeological chemistry. Chungara, 39(2): 235-257.

Tótora Da-Gloria, P.J., W. Neves, M.A. Costa y Bartolomucci. 2011. Nonspecific infectious diseases in prehistoric San Pedro de Atacama, northern Chile. Chungara,43(1): 135-146.

Uribe, M., C. Agüero. 2001. Alfarería, textiles y la integración del Norte Grande de Chile a Tiwanaku. Boletín de Arqueología, 5: 397- 426. PUCP.

Varela, H., J. Cocilovo y M.A. Costa. 1990. El dimorfismo sexual en la población prehistórica de San Pedro de Atacama, Chile. Chungara, 24/25:159-166.

Waldron,T. 2009.Paleopathology. Cambridge University press, New York.

Weiss, E., R. Jurmain. 2007. Osteoarthritis revisited: A contemporary review of aetiology. International Journal of Osteoarchaeology, 17: 437-450.

Yousuf, A. S. 1978. New knowledge of osteoarthrosis. Journal Clinic Pathology, 31 (12): 191-199.

Zhang Y., D. Hunter, M. Nevitt, L. Xu, J. Niu, L-Y Lui, W. Yu, P. Aliabadi y D. Felson. 2004. Association of squatting with increase prevalence of radiographic tibiofemoral knee osteoarthritis. Arthritis and Rheumatism, 50 (4): 1187-1192. 